\title{
Terapia Sistémica e Institución Pública. De la teoría a la práctica en la recogida de la Demanda
}

RESUMEN: Se describe la estructura de la entrevista que nos permita conocer el mapa relacional actual y pasado del individuo, así como su contexto significativo y contrato terapéutico.

PALABRAS CLAVE: Terapia Sistémica, Terapia familia, Primera entrevista, Recogida demanda, Análisis demanda.
SUMMARY: The structure of the interview is described, that the option to know contribute us the present relational map and past of the individual and the significant context and therapeutic contract.

KEYWORDS: Therapy Systemic, Therapy Family, First interview, Collected demand, Analysis demands.

\section{Introducción}

«El hombre es el hombre más sus circunstancias» (Ortega y Gasset). Una buena manera de iniciar esta reflexión es con un aforismo que defina la forma en la que estudio al individuo: con una visión binocular y en tres dimensiones; me refiero al individuo en su mundo relacional, y con un reflejo en el presente, de su pasado, siendo el promotor de su futuro.

La primera cuestión que surge sería si desde la Institución Pública se puede intervenir adecuadamente con un paradigma en psicoterapia. Las respuestas son en su mayoría problemas o bien resistencias, ¿de la institución o de los profesionales? No tenemos el tiempo que se precisa en cada intervención, no podemos preparar la sesión previamente, ni la periodicidad de las citas es la pertinente, desconozco la demanda del paciente, desconozco los miembros que van a acudir a la consulta, a ello añado que me puedan interrumpir durante la sesión, que el o los demandantes no acudan a su hora, por lo que el tiempo asignado se reduzca, no existe coterapia, etc.

Desde mi trabajo como psiquiatra, y en los últimos años en consultas externas, atendiendo a adultos, he tenido la posibilidad de compartir muchos relatos con los pacientes, por lo que el objetivo sería exponer la forma de recoger la información que clarifique al profesional encargado del caso, de qué hipótesis partimos y qué técnicas serían las más útiles para alcanzar las metas propuestas.

Al utilizar la palabra paciente, no quedan excluidos otros miembros que lo acompañen y que intervengan en la recogida de la información, hace referencia a que en la mayoría de las ocasiones se realiza con el paciente, o bien con subsistemas.

\section{Bases teóricas}

Casi de forma automática cada uno de nosotros va instaurando una forma de comunicación con el paciente en los primeros encuentros, que conforman un estilo y que suele seguir, como en nuestras relaciones, una secuencia redundante. 
No me gusta hablar de escuelas, entendidas como una doctrina inmóvil a seguir, sino más bien de modelos o líneas teóricas que dentro de la terapia sistémica articulan los primeros encuentros con el paciente, sin sentirme encorsetada en una sola de ellas, ni prisionera de reglas que rotulen a los demandantes.

Todos somos conocedores de la importancia que tiene el primer contacto; de él se derivan no sólo las reglas de la relación, tanto implícitas como veladas, sino una epistemología compartida de su patología, una forma de abordaje y los objetivos a perseguir. Incluso podemos considerar que el fracaso del encuentro supone casi siempre el fracaso del tratamiento (1).

De forma breve voy a nombrar modelos y autores que más han influido en mi manera de estructurar los primeros encuentros con los pacientes. La división por enfoques atiende no a una estructura preestablecida, sino a una agrupación didáctica.

Incluido en el Enfoque Psicoanalítico, resaltar a M. Bowen en su estudio de la individualidad de cada miembro, dentro de un sistema familiar con características propias. Conceptos como Triángulo emocional, en el que defiende que la triada es la base de todo sistema relacional y Transmisión multigeneracional, que hace referencia a la presencia del pasado en el presente, vehiculizado a través del proceso emocional de la familia, y de cada miembro individualmente.

Otro autor perteneciente a esta línea, Boszormenyi-Nagy (3), habla de Un libro mayor de cuentas, que expresa el balance transgeneracional entre obligaciones y méritos, determinadas por las características psicológicas de cada miembro y las reglas del sistema.

Un segundo enfoque, el Comunicacional o Mixto, ofrece una posición intermedia. El individuo se contempla como un subsistema, con su propia estructura psicológica, en relación con los otros subsistemas.

Se incluye el denominado Modelo Estructural fundado por S. Minuchin (4), según el cual, la adecuada funcionalidad de la familia dependerá de su estructura, que se basa en los límites, alianzas y jerarquías entre los subsistemas.

En el Enfoque Sistémico, alejado del planteamiento psicoanalítico, el individuo es entendido en interacción con otros miembros de un sistema. G. Bateson (5), plantea un nuevo paradigma, partiendo de la Teoría de la Comunicación, la Cibernética y la Teoría General de los Sistemas.

En esta línea se encuentra el Modelo estratégico, su principal autor, J. Haley (6), parte del supuesto que el cambio del paciente se produce mediante modificación directiva del tipo de soluciones indeseadas, sustituyéndola por otra más exitosa. Hemos de destacar, en este enfoque, el modelo con el que más afinidad comparto, la Escuela de Milán, con M. Selvini y G. Prata como principales iniciadoras (7). 
Según la afirmación de C. Rojero y T. Suárez (1981): «el trabajo en la búsqueda de estrategias para el cambio de las reglas familiares disfuncionales y el trabajo transgeneracional son, sin duda, las dos grandes características del tratamiento» (8). Encontramos su singularidad en la introducción de la «ficha telefónica». Establece para la coordinación de las sesiones terapéuticas tres directrices (1): la elaboración de hipótesis, la circularidad y la neutralidad. Introduce el concepto de «juego», en el cual cada miembro posee unas estrategias dentro del sistema para alcanzar sus objetivos. El paciente atiende al «principio de competencia», según el cual da opiniones en igualdad de condiciones que el resto de miembros.

Con la evolución del pensamiento sistémico y el desarrollo de la segunda cibernética, aparece el enfoque constructivista, más cercana al cognitivismo y al estudio del individuo. Según este modelo creamos nuestra propia realidad dentro del contexto en el que vivimos (9). Se produce una ruptura tajante de la asimetría entre el terapeuta y el paciente, este primero pierde su posición de experto, el cliente toma el control sobre su vida y no se indaga la etiología de los síntomas, sino los mecanismos que los mantienen.

Dentro de esta línea nombrar la terapia centrada en soluciones, su iniciador, De Shazer, se distancia de la pregunta por qué o para qué, para centrarse en las soluciones (11). También reseñar la formulación de la «conversación externalizadora» de M. White (12), definida como una conversación en la que las personas relatan de qué manera el problema ha estado afectando su vida y sus relaciones. El término «excepciones», utilizado por De Shazer, sería similar al de «acontecimientos extraordinarios» de M. White, serían acontecimientos que formula el paciente sobre el cuidado de sí mismo y su capacidad de adaptación, ambos son útiles para concretar objetivos y dar temporalidad a la terapia.

De forma general, podemos decir que tanto el modelo estructural como el estratégico y el constructivismo, comparten el trabajo en el aquí y ahora, se concentran en la ineficacia de las soluciones intentadas, así como una mayor brevedad de la terapia, a diferencia de la Escuela de Milán que estudia el «juego» en curso y analiza el funcionamiento a lo largo del ciclo familiar.

\section{Marcadores de contexto: atrapados en el juego institucional}

El contexto terapéutico en la Institución Sanitaria pública va a estar marcado por reglas que nos informan del marco de referencia. Las implícitas, en su mayoría corresponden a las garantías de asistencia (Ley General de Sanidad, 1986) y otras acordadas, que son más específicas de la relación médico-paciente. 
Resaltaría las reglas que nos atrapan como terapeutas y son aquellas en las que coincide el sistema sanitario, social e individual:

1. Según el modelo sanitario vigente, la visión de la enfermedad es lineal, causa-efecto, y a la vez dicotómica: enfermedad / salud, padecimiento / curación. Etimológicamente, terapeuta es el que cura.

El paciente asiste a la consulta a la espera de que desaparezcan los síntomas, pero a la vez en una actitud de ignorancia y desvalimiento, posicionando la sabiduría y la técnica al terapeuta. Uno de los primeros aspectos a trabajar en el primer encuentro sería que el paciente no nos posicione como el director de su propia vida. Su devenir histórico lo conforma él y sus relaciones, tendríamos que ser capaces de transmitir que somos intervinientes temporales en sus vidas, el mayor o menor éxito en las metas que se marquen es un continuo, no sólo en el aquí y ahora, sino también en la construcción futura.

2. Una de las paradojas a la que nos enfrentamos es la del tiempo, a la vez se ha convertido en un mito, la queja que más se oye en la sanidad pública es «no me dan el tiempo suficiente para atender al paciente», y, por el contrario, tenemos toda una vida por delante el paciente y el terapeuta, ya que una condición básica de nuestro Sistema Sanitario es que se le garantiza la asistencia en su padecimiento.

Debemos plantearnos la adecuada utilización del tiempo, ¿qué hacer con el exceso de éste?, no con el defecto.

Por supuesto, tenemos que entender que esta regla del Sistema Sanitario Público, el paciente puede utilizarla de la manera que menos energía le suponga, incluyéndonos entre sus relaciones significativas, pero entonces ¿seremos agentes de cambio o bien ejerceremos la función de «mantener más de lo mismo»? No podemos escapar de este condicionamiento, pero sí utilizarlo bien, complementando el tiempo psicológico del paciente con el tiempo terapéutico, que éste primero no sea indeterminado, sino llegando a un contrato de trabajo, acercándonos a concretar número de encuentros, frecuencia, objetivos, etc.

Habrá pacientes que busquen un homeostato en su terapeuta, que le acompañe un largo período de tiempo, en estos casos, nos podemos valer de los acontecimientos propios del ciclo vital y de los no normativos (extra-terapéuticos) en los que podemos realizar un imput que favorezca el cambio.

Revisando la literatura de investigación sobre los factores que explican el cambio en psicoterapia se señala que el $40 \%$ del cambio se atribuye a factores extra-terapéuticos, como variables del cliente o del ambiente; $15 \%$ al efecto placebo como la esperanza en la terapia; $15 \%$ a las técnicas; y el $30 \%$ a la relación terapéutica (13). Estos datos, lejos de favorecer el pesimismo en psicoterapia, creo que la sitúa en un lugar privilegiado para el cambio, ya que las técnicas utilizadas van a influir, tanto en la relación médico-paciente como en el inicio y mantenimiento del cambio ante los factores exteriores. 
Por supuesto existen patologías que van a precisar de un seguimiento continuado, no sólo por su gravedad clínica, sino por su necesidad de acompañamiento, pero en estos casos el trabajo terapéutico iría en la línea de dotar al paciente de estrategias propias que faciliten su autonomía, es decir, su competencia, el objetivo último no sería su alta, sino un seguimiento más espaciado.

3. Esta paradoja del tiempo, va asociada a otra regla del Sistema Sanitario, según la Ley General de Sanidad, en su artículo 6, punto 4, dice: «Se garantiza la asistencia a todos los casos de pérdida de salud», de manera que no cabría la indicación de «no tratamiento» (14). Si se utiliza como técnica paradójica se incluye en las estrategias para el cambio, por lo que puede ser útil dejando un tiempo libre de intervención, y reiniciar con posterioridad los encuentros, si éste no ha modificado el funcionamiento familiar, introduciríamos otras técnicas.

El problema lo encuentro cuando se utiliza como forma de «delegar la responsabilidad» a otro terapeuta. En este caso, se puede convertir en útil para el individuo o familia, siempre que la derivación se realice con coordinación previa, con análisis del caso, resistencias y objetivos; de lo contrario dañamos, al entender el demandante que «su caso no tiene solución» y, en muchos casos, implica una segunda conclusión: «necesitaré siempre tratamiento».

4. Suele acudir a consulta el individuo a solas, o acompañado de uno o varios miembros de la familia. Llegan en un momento de desorganización; la petición es volver al estado previo a la aparición del síntoma, si éste lo entendemos como expresión de patrones de relación redundante que no han dado solución, buscaríamos no volver al statu quo, no quedar incluidos en el juego familiar, sino nuevas estrategias que cambien el funcionamiento del sistema. En la medida en que cada vez llegan más patologías «benignas» a consulta, más tenemos que cuidar este aspecto.

Tenemos que darnos cuenta de que el paciente o la familia que nos dice «qué contentos estamos con usted», en algunos casos, es consecuencia de haber sido incluidos en su sistema relacional como agentes perpetuadores de su disfunción relacional.

Nos preguntaríamos, si ellos están satisfechos, ¿por qué modificar nuestra actuación? Desconozco la respuesta de cada profesional, pero lo que he constatado es que las consultas quedan repletas de casos más benignos, y tanto éstos como los más graves mantienen la clínica, algo más atenuada, no se espacian las citas y ante los momentos de crisis, no movilizan sus propios recursos, acuden a su consultor.

\section{Fases de la recogida de la Demanda: de la teoría a la práctica}

La recogida de la demanda que aporte la información para la elaboración de hipótesis, suelo realizarla en una o dos intervenciones, el tiempo viene acordado 
por el contrato-gestión del hospital. La primera sería de 45 minutos, y si se utiliza una segunda, de 20 minutos.

Si realizamos un análisis comparativo entre la teoría sistémica del «primer contacto», especialmente las directrices de la Escuela de Milán y las diferencias con la actuación en la Institución Sanitaria, hay que distinguir tres fases: la fase previa al contacto, en ella se llevaría a cabo la «Ficha telefónica», que es como una base de datos en la que el foco no se pone sólo en el síntoma, sino también en el contexto; se indaga la explicación que da el solicitante del síntoma, se recogen los datos de la familia nuclear y extensa y se da la fecha, y miembros que se citan en la primera sesión. Incluye el análisis del derivante, al que se otorga un gran valor, ya que la información previa que posea el paciente de nosotros puede facilitar, entorpecer o destruir nuestra intervención. De esta ficha el o los terapeutas inician la elaboración de hipótesis (15). En la segunda fase o de acogida, se inicia el contacto, que conduce a la explicitación de las condiciones que lleven a compartir un espacio terapéutico, y, la tercera o fase de acoplamiento, hace referencia a las acciones del terapeuta tendientes directamente a relacionarse con los miembros de la familia, y a las adaptaciones del terapeuta tendientes a lograr la alianza.

En nuestro trabajo diario se suman las tres fases, y se complementan en la primera entrevista, sin pausa entre ellas y no siendo posible coterapia.

Veamos las particularidades que conviene resaltar:

A. En la tramitación de la demanda actúa el modelo vigente de atención sanitaria, con las especificidades de la gestión de cada hospital. La derivación suele realizarla el médico de cabecera, pero también puede solicitarla psicología u otro médico especialista a través de una interconsulta. La información del paciente quedará reflejada en la hoja ya formalizada para ello. Por regla general la descripción de la petición se realiza en dos o tres líneas, que ofrecen datos de manera muy general sobre el cuadro sindrómico que padece el paciente. Los datos suelen ser síntomas y signos clínicos, con algún aspecto de tipo psicosocial. Un error destacable es que solemos obviar el estudio del derivante, damos por sentado que será un derivante «desinterado» o «partícipe», que no interfiera en nuestra intervención, no pensamos que esté «involucrado» o sea «antagonista» y frene o descalifique las actuaciones (17).

Pienso que preguntas como: ¿quién le envía?, ¿por qué cree que le han derivado? ¿le han dado alguna información previa del Servicio o los profesionales que lo integran? Con poco tiempo nos ofrecerá información valiosa. Piensen por ejemplo, en una derivación dirigida específicamente a uno de nosotros, en la atención de un paciente derivado tras petición de cambio de terapeuta, etc.

B. Sobre la fase de acogida y acoplamiento, destacar: 
Partimos de dos premisas: «La objetividad es imposible de alcanzar» $(\mathrm{H}$. Foerster, 18) $y$ «no podemos observar algo sin modificarlo» (19). Nuestra forma de saludarlos, invitarle a que tomen asiento, entonación, mirada, vestimenta, decoración del despacho son ejemplos de que en la interacción es imposible no comunicar, todos los miembros que intervienen van a dar especificidad a ese sistema creado, de manera que la conducta y expresión de cada uno influye y es influida por todos los otros. ¿Cuidamos estos aspectos?

¿A quién hacemos pasar? El paciente designado puede venir solo, pero en muchas ocasiones, le acompañan uno o más miembros. Si no explicitamos nada suele pasar solo el individuo, o bien, nos solicitan si puede pasar alguien más. Cuando acuden con acompañantes, la entrevista no se tiene porqué alargar, y, por el contrario, es más rica en datos. Creo que para obtener mayor información, la forma más útil sería preguntar al paciente con qué miembros ha acudido y sólo si él está de acuerdo, invitar a los que deseen pasar. Si tengo dudas de quién debe participar, le pregunto al paciente que él los elija, y posteriormente indago las razones de esa elección.

El posicionar al paciente con capacidad de decidir, atiende a otro principio, que es el de Competencia, va a ser partícipe de su proceso de salud. A través de esta secuencia de actuaciones estoy rompiendo la linealidad: cuidador/cuidado, guía/seguidor, activo/pasivo, sabiduría/desconocimiento.

En la relación terapeuta-paciente el concepto de metacomplementariedad es un término utilizado por Haley, denomina la posición en que se coloca el terapeuta para poder elegir entre tres posiciones frente al paciente: superioridad, igualdad, inferioridad. Si entendemos el cambio como promoción de recursos del paciente se pueden utilizar todas ellas, evitando colocarse en una posición superior o directiva continuadamente.

En el caso de efectuarse la entrevista con más de un individuo, si no favorecemos la participación de éstos en la sesión, no habremos cambiado la epistemología de la que parten, de ahí que, durante la conducción de la entrevista, las preguntas sean circulares, a través de ellas nos ayudamos a ensamblar la visión relacional del síntoma.

El terapeuta debe demostrar empatía y capacidad de adaptación a contextos cambiantes, con capacidad de autocrítica, en caso de errores, o malos entendidos, así como utilizar la ironía y el humor para tratar aspectos especialmente dolorosos.

Ante la nueva forma de recoger los datos, algún miembro invitado puede sentirse señalado o culpabilizado, debe quedar clarificado que la búsqueda no es de culpables e inocentes, sino de entender cómo, de forma no deliberada, ha sido partícipe de una secuencia de interacciones que ha llevado a la situación de crisis. 
Se transmite que las informaciones relevantes se deben compartir con el grupo; es una manera implícita de no aceptar coaliciones, como forma de mantener la neutralidad (20). Pensemos en las múltiples ocasiones que un miembro de la familia nos da información, solicitándonos el secreto compartido.

Debe intervenirse respetando a las personas y al sistema familiar en su estilo, ideología y valores. Un desafío frontal a cualquiera de estos puntos lleva al fracaso o a un rechazo que provocaría resistencias.

Hemos de adaptarnos al momento psicológico del individuo o la familia, respetando su tiempo, ya que es posible que en una o dos entrevistas no se establezca una relación de confianza suficiente, omitiendo información crucial o rechazando nuestra forma de abordar el problema.

La gravedad del caso la entiendo como distanciamiento de sus proyectos previos, y la mido por su deterioro personal, social y laboral. De esta forma suele coincidir diagnóstico clínico y diagnóstico sistémico. Utilizar exclusivamente el diagnóstico sindrómico, en muchos casos, puede servir en bandeja no las resistencias del paciente, sino del terapeuta: «Tiene usted un trastorno de personalidad, su caso es crónico».

La coherencia entre la conducción y conclusión de estos primeros encuentros debe basarse en la información obtenida, si no hemos podido realizar o verificar hipótesis, una conclusión inespecífica no posee potencial de cambio (21).

Es muy difícil separar la etapa de evaluación de la etapa terapéutica. Como dice Minuchin, diagnóstico y tratamiento permanecen inseparables a lo largo de todo el proceso terapéutico.

Todos los aspectos antes reseñados, facilitan el ensamblaje del espacio cognitivo, emocional y conductual del paciente o la familia, siendo esta unión el vehículo para el cambio.

\section{Análisis de la demanda}

La flexibilidad del terapeuta para adaptarse al sistema con el que va a trabajar es un principio básico, de ahí que las singularidades que comento a continuación se deben acomodar para obtener la información pertinente, sin dejarnos llevar por datos irrelevantes.

Todos los datos recogidos van conformando en el terapeuta un doble diagnóstico, el clínico y el sistémico. Sólo en un pequeño porcentaje de casos serán candidatos de concluir con una psicoterapia individual, de pareja o de familia, aspecto que no amplío, ya que no es el tema de esta intervención.

Destaco como directrices: 
ORIGINALES Y REVISIONES

1) La recogida de la demanda no lleva un orden estricto, pero sí preestablecido, adaptándose al relato del paciente.

2) La observación de las interacciones tanto digital como analógica.

3) Se contemplan los sucesos tensionantes ocurridos transgeneracionalmente, así como los recursos puestos en práctica.

4) Se indaga la crisis en el aquí y ahora, inmersa en el ciclo vital del individuo y en su contexto.

5) Énfasis en la relación, pero valorando la individualidad a través del relato construido por cada individuo.

6) En la mayoría de ocasiones se abordará la recogida de información con el paciente a solas, o bien con los miembros que acudan.

\section{Estructura de la entrevista}

Se iniciaría preguntando por el derivante: ¿de quién ha partido la petición de esta consulta?, ¿sale la razón de esta derivación?, ¿le han realizado comentarios del servicio o del personal?

Se suele preguntar: ¿qué le ocurre? ¿cuál es su problema? De esta manera se está definiendo el contexto terapéutico en el que se hablará de problemas. En cambio si la pregunta es: ¿en qué le puedo ayudar? Introducimos desde el comienzo un doble mensaje, conversar sobre sus dificultades y sobre las soluciones a compartir.

Seguidamente, se solicita que nos comenten, empezando por el paciente, la conducta sintomática. Se pregunta ¿cuándo empezaron los síntomas?, ¿a qué lo atribuyen?, ¿a qué otros profesionales se ha consultado?, ¿por qué consultan «ahora»?

Junto a la descripción sintomática, y con la finalidad de llegar a un diagnóstico sistémico, se realizan hipótesis que no tienen valor de verdad, sino de utilidad, nos proporcionan información y posibilidad de cambio. Se ordena a lo largo de tres niveles que se entremezclan: elaboración del genograma, exploración de áreas concretas y observación de las interacciones durante la entrevista.

A través del genograma, los miembros en consulta, van introduciéndose en otra epistemología, de forma gráfica van observando cómo el tiempo ha ido configurando un mapa de relaciones; incluso suelen señalar para explicarnos datos. Deben recogerse tres generaciones; es útil en procesos leves, e indispensable en los casos graves. Obtendremos datos sobre: quiénes están implicados en el problema; funcionamiento de diada conyugal; subsistema parento-filial y la fratría; análisis de alianzas, coaliciones, triangulaciones, límites intergeneracionales, lími- 
tes con el exterior; pautas multigeneracionales a través de sucesos significativos del pasado como muertes, nacimientos, jubilación, etc. Creencias, mitos de la familia. Juego relacional en curso.

Las preguntas se pueden categorizar en (22):

Lineales: ¿Quién lo hizo? ¿Cuándo?

Circulares: ¿Qué hace su hijo cuando usted discute con su mujer?

Estratégicas: ¿Cuándo va a dejar de comportarse como un niño malcriado?

Reflexivas: Estas últimas son especialmente útiles en trabajo individual, como las utilizadas en psicoterapia basada en soluciones (11): ¿qué cosas notaría si al despertarse no existiera el problema? ¿qué haría, qué sentiría, qué pensaría si no existiese el problema? ¿Qué ha hecho, pensado o sentido para solucionar el problema? ¿qué le ha sido favorable? ¿qué no ha servido para mejorar? Así como, por ejemplo, no es extraño encontrarnos con este comentario por parte del paciente: ¿me han dicho que tengo una depresión crónica y que no tiene curación? Cuando el diagnóstico clínico invade la identidad del sujeto, y la recogida es individual, son útiles preguntas que introducen una visión externalizadora (12) como: ¿de qué modo el problema afecta tu vida?, ¿qué le está haciendo a tus amistades? ¿cómo interfiere en la relación con tus padres?

La clarificación es una técnica que puede utilizarse en cualquier fase de la entrevista, con intención de comprobar que se ha comprendido correctamente el mensaje y como estrategia para resaltar comentarios cruciales, que se manejarán posteriormente en la conclusión.

En la parte final de la entrevista se puede utilizar la recapitulación, que aglutina los temas, las redundancias o las consistencias de la comunicación de la familia. En muchos casos esta técnica no es puramente un resumen de la entrevista, sino aperturas para contrastar las hipótesis. Se debe distinguir este concepto del de conclusión (23) que consiste en la información obtenida en la entrevista, de aquellas hipótesis que han sido verificadas.

Si se utiliza una segunda entrevista, los cambios favorables obtenidos por el paciente son elogiados por el terapeuta, redundando en la participación activa del demandante (11).

Al finalizar el análisis de la demanda es importante obtener del individuo o del susbsistema que ha participado:

1) Un mapa relacional de la situación de crisis actual, entendida dentro de estrategias individuales en torno al problema, enmarcado en un acontecer pasado de relaciones significativas, que dan la especificidad del individuo.

2) Una formulación suficientemente clara de objetivos alcanzables durante el proceso de tratamiento. 
ORIGINALES Y REVISIONES

3) Si se puede, fijar otros elementos del contrato terapéutico como son: elección del tipo de terapia, subsistema elegido, duración, frecuencia y número de sesiones.

A continuación expongo un caso, elegido al azar, donde sólo se recogen extractos de la entrevista. Sería destacable que acude como caso nuevo y que posee una larga historia en salud mental, ha sido vista por varios psicólogos y psiquiatras, característica muy común en la asistencia pública. Corresponde a los casos en los que no se establece contrato terapéutico y la regla implícita de la relación es la no temporalidad en la atención, es un seguimiento indefinido. Los datos expuestos son de una entrevista de 45 minutos.

En el estudio de la historia, existen datos desde el año 2000 al 2004, desde entonces hasta la derivación (mayo, 2006), a pesar de que estaba siendo seguida, las anotaciones no están en su dossier. Resumo datos relevantes y su descripción para entender la nueva recogida, no la nueva Demanda.

Presentó un cuadro depresivo a los 22 años (1994), fue tratada varios meses por psiquiatra privado, estando asintomática hasta diciembre de 2000 (27 años) que solicita atención, siendo diagnosticada de trastorno por angustia con agorafobia y se asocia a estrés laboral. Presenta dos ingresos en medicina interna por 5-10 deposiciones diarias e importante pérdida de peso, coincidiendo en el diagnóstico de colon irritable y síndrome ansioso-depresivo. Aparecen datos psicobiográficos: está casada, tiene hijos, es la mayor de tres hermanas, la segunda posee retraso mental por meningoencefalitis; su madre fue atropellada y está inválida. Se produce mejoría con tratamiento, dándosele de alta en 2002, y vuelve a solicitar asistencia psiquiátrica en 2004 que ha seguido hasta la actualidad, en esta última petición, destacar el nacimiento de su hijo hacía 10 días. Se incorpora abordaje psicológico desde marzo de 2006, en el que se anota: «cualquier pequeña cosa le agobia y deja de hacerlo, pues la incertidumbre le paraliza, evita hacer más cosas para poder atender a su hijo, le gustaría trabajar». Poco después de la entrevista en la que aparecen estos datos su padre ingresó en UCI tras parada cardio-respiratoria. La recogida de la Demanda la efectuó en mayo de 2006.

Antes de pasar a la paciente inicio el proceso de hipotetización, iré a buscar nudos relacionales en los que apareció clínica. Parece estar sobreimplicada en los cuidados de la familia de origen, con dificultades en su proceso de separaciónindividuación, tanto antes de casarse, como después, a la vez habría que confirmar una frágil delimitación del subsistema parento-filial, con parentalización de la paciente, teniendo una madre «inválida» y una hermana con «retraso mental». Ante el nacimiento de su hijo, tiene una nueva crisis, en la misma línea de hipotetización, reaparece el dilema sobre su lealtad hacia su esposo e hijo, y a la vez con 
los cuidados que precisa su familia de origen. Si nos vamos atrás en el tiempo, ¿el rol de cuidadora se estableció desde la infancia, siendo ella la primogénita, con una hermana enferma que nació dos años después? ¿qué papel jugaron los padres ante el diagnóstico de la hermana? ¿desde cuándo data la debilidad materna?

Acude sola con un carrito de bebé vacío. En este caso, el derivante, la ha informado que al modificarse su destino laboral, debe cambiar de psiquiatra, para la paciente esta situación es habitual, y su predisposición es buena, ya que le han ayudado en su proceso.

T: ¿En qué te puedo ayudar?

P: No sé, me han enviado para que me siga viendo, ahora tomo poca medicación, mi padre tuvo una muerte súbita en marzo, y por ahora estoy bien.

T: Tras todo el tiempo que llevas en tratamiento, ¿a qué atribuyes tu enfermedad?

P: Soy débil, la causa está en mí.

Se inicia la recogida del genograma, y se van confirmando y refutando hipótesis en un relato conjunto con la paciente.

T: ¿Cuéntame qué ocurrió cuando empezaron tus síntomas en 1994?

P: Vivíamos en un pueblo, trabajaba en una fábrica de calzado, allí no había nada, pensé en venirme a Albacete.

T: ¿Qué opinó tu padre y tu madre?

P: No me dijeron nada, entonces mi hermana tenía pocas crisis epilépticas, la otra trabajaba, era un buen momento.

T: ¿Un buen momento?

P: Bueno, ¿por qué no podía buscar lo que yo quería?

T: Empezaste a encontrarte mal.

P: Sí, estaba preocupada por cómo funcionaría mi casa, pero yo no podía seguir en aquel pueblo... Mi hermana pequeña también quería venirse a Albacete, fueron hablando del tema y unos dos años después se vinieron.

T: ¿Parece que lo pasaste mal cuando tuviste las diarreas?

P: Francamente sí, pensaba que tenía algo malo, perdí mucho peso.

T: ¿Pudiste encontrar explicación a estas diarreas?

P: No, nunca supe porqué me ocurrió, me dijeron que era de nervios.

T: ¿Parece que coincide en el tiempo con el accidente de tu madre?

P: Tiene razón, nunca me había dado cuenta de esto, fue un momento muy difícil... tuvo varias intervenciones, después complicaciones, y juicios... (se van 
intercalando preguntas que incluyen a todos los miembros)... mi padre era fuerte, él ayudó mucho... si no hubiera sido por él...; mi hermana estaba trabajando, ella también ayudaba, pero sobre todo mi padre y yo..., menos mal que mi marido es muy comprensivo y siempre ha estado a mi lado.

T: ¿Qué recuerdas de la ayuda que necesitaste en el 2004?, ¿tu hijo había nacido hacía poco?

P: No dilataba en el parto, tuvieron que utilizar fórceps, pensé que se iba a morir, sólo le pude dar pecho dos semanas, y con la leche tuvo intolerancia (se intercalan preguntas del terapeuta)... mis padres me ayudaron... pero yo siempre he sabido que mi madre necesitaba ser cuidada, mi padre y yo hemos sido los fuertes... también recuerdo que mi hermana empezó a tener más crisis... fueron momentos difíciles.

T: ¿Qué te ha ocurrido ahora?

P: Todo iba bien, estaba pensando en volver a trabajar... pero ahora mi casa es un infierno (se refiere a la casa de sus padres), desde que mi padre tuvo la muerte súbita y mi hermana que sigue con las crisis, estoy entre la casa de mis padres y la mía... ahora me ayudo de mi marido y de mi hermana menor.

T: ¿De qué te ha servido el tratamiento estos años?

P: He aprendido a no pensar tonterías, a buscar el lado bueno, a disfrutar de mi peque... porque estos tres últimos meses lo he visto poco... no racionalizar las cosas, me veo ahí intentando remontar.

T: ¿Has pensado qué otras cosas puedes hacer para remontar? Si un día te despertaras y fueras fuerte ¿qué harías?, ¿con quién cuentas para lograr tus objetivos? Te defines como una persona débil, ¿lo sigues creyendo después de todo el relato de superación de dificultades?, ¿de qué manera te está afectando el problema de ser la que tira de las riendas de tus padres? Seguiremos hablando de todo ello.

\section{BIBLIOGRAFÍA}

(1) SuÁrez, T.; Rojero, C. F., «La técnica de la Terapia Familiar Sistémica y el problema de la formación», en Paradigma Sistémico y Terapia de Familia, Madrid, AEN, 1983.

(2) Bowen, M., De la familia al individuo, Barcelona, Paidós, 1996.

(3) Boszormenyi-Nagy, I., Lealtades invisibles, Buenos Aires, Amorrortu, 1983.

(4) Minuchin, S., Familias y terapia familiar, Barcelona, Gedisa, 1979.

(5) Bateson, G., Pasos hacia una ecología de la mente, Buenos Aires, Planeta, 1991.

(6) Haley, J., Tratamiento de la Familia, Barcelona, Toray, 1974.

(7) Selvini, M., y otros, Paradoja y contraparadoja, Barcelona, Paidós, 1991.

(8) RoJero, C. F.; SuÁREZ, T., «Prevención: encrucijada de intervenciones sistémicas», Rev. de Psicoterapia y Psicosomática, 1981, Nueva Época, n. ${ }^{\circ} 3$, pp. 7-23.

(9) Watzlawick, P., La realidad inventada, Buenos Aires, Gedisa, 1988. 
(10) Anderson, H.; Goolishian, H., La terapia como construcción social, Barcelona, Paidós, 1996.

(11) De Shazer, S., Claves en Psicoterapia Breve, Barcelona, Gedisa, 2004.

(12) White, M., Reescribir la vida, Barcelona, Gedisa, 2002.

(13) EsCudERo, V., «Simposio Psicología, Investigación y Terapia Familiar. ¿Hacia dónde hay que ir?», Cuadernos de Terapia Familiar, 2004, n. ${ }^{\circ}$ 56-57, pp. 37-38.

(14) RoJero, C. F.; SuÁREz, T., «Marca de contexto y conclusión de la primera entrevista», Revista de la AEN, 1984, n. ${ }^{\circ} 11$, pp. 363-372.

(15) SElvinI, M., y otros, «El problema de la persona que sugiere la Terapia Familiar», en Crónica de una investigación, Barcelona, Paidós, 1990.

(16) Di Blasco, P.; Sischer, J. M.; PrATA, G., «La ficha telefónica: piedra angular de la primera entrevista con la familia», Revista clínica y Análisis Grupal, 1988, XII, n. ${ }^{\circ} 47$.

(17) Campanini, A.; LupPI, F., Servicio Social y Modelo Sistémico. Una perspectiva para la práctica cotidiana, Barcelona, Paidós, 1991.

(18) Foerster, H., Las semillas de la cibernética, Barcelona, Gedisa, 2005.

(19) ZuKaV, G., La danza de los maestros del Wu Li, Barcelona, Plaza y Janés, 1991.

(20) Rojero, C. F., y otros, «Hacia un programa de Salud Metal Comunitario: aspectos de un modelo sistémico de organización e intervención», Revista de la AEN, 1986, Vol. VI, n. ${ }^{\circ} 18$, pp. 438-452.

(21) Rojero, C. F.; SuÁRez, T.; VÁzQuez, P., «La entrevista sistémica: de la conducción a la conclusión», Rev. Psicoterapia y Psicosomática, 1983, Nueva Época, n. ${ }^{\circ} 7$.

(22) NAVArro, J., Técnicas y programas en terapia familiar, Barcelona, Paidós, 1992.

(23) RoJero, C. F.; SuÁrez, T.; VÁzQuez, P., «La entrevista sistémica: de la conducción a la conclusión», Rev. Psicoterapia y Psicosomática, 1983, Nueva Época, n. ${ }^{\circ} 7$.

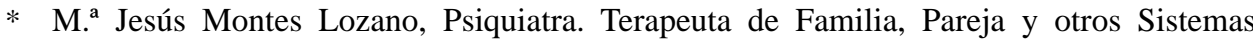
Humanos. Complejo Hospitalario Universitario de Albacete. Servicio de Salud Mental. Correspondencia: M. ${ }^{a}$ Jesús Montes Lozano. Paseo Cuba, n. ${ }^{\circ}$ 3, 4. ${ }^{\circ}$ A. Albacete, 02001. e-mail:mjmontes@sescam.jccm.es 